\title{
Diffuse myelitis in a 9-month-old infant: case report and review of the literature
}

\author{
O. Hüdaoglu, ${ }^{1}$ U. Yis, ${ }^{1}$ S. Kurul, ${ }^{1}$ H. Çakmakçi, ${ }^{2}$ M. Saygi ${ }^{3}$ and E. Dirik ${ }^{1}$
}

\section{Introduction}

Acute transverse myelitis is a rare but severe inflammatory demyelinating disorder that usually involves both sensory and motor tracts of the spinal cord [1]. It is characterized by rapid onset of paraplegia or tetraplegia, loss of sensation and sphincter disturbance, and in rare cases it can cause respiratory insufficiency. This disease occurs commonly among adults and rarely in the paediatric population, especially children under 2 years of age [2,3]. The exact pathophysiological mechanisms and trigger factors that result in neural injury are not well understood. However, recent studies point to a variety of humoral and cellular immune derangements that potentially result in neuronal injury and demyelination $[4,5]$.

We report a case of a 9-month-old boy who presented with a 1 month evolving history of progressive generalized weakness.

\section{Case report}

A 9-month-old male patient was admitted to the paediatric neurology department because of progressive generalized weakness which had started 1 month before. There were no important features in his prenatal, natal and postnatal history, except an upper respiratory tract infection 3 weeks before the clinical onset of symptoms. The family history was unremarkable.

On physical examination he was oriented to person and his vital signs were within normal limits. He was not able to move any limbs, muscle tone was decreased and deep tendon reflexes were absent in his upper and lower extremities. He was crying in response to pain, but unable to move his limbs. Abdominal, cremasteric and anal reflexes could not be obtained. Babinski sign was absent. Cranial nerves were intact. The remainder of the physical examination was normal.

In order to rule out spinal cord pathology, craniospinal magnetic resonance imaging (MRI) was obtained immediately. On T2 weighted images, a diffuse, symmetrical increased signal within the whole spinal cord was observed. Lesions showed no contrast enhancement (Figure 1). Contrastenhanced MRI angiography demonstrated normal vascular structures. No other abnormality was detected. Cranial MRI revealed no abnormality.

On laboratory examinations, the haematological and biochemical parameters for serum were within normal ranges. Cerebrospinal fluid protein was $15.8 \mathrm{mg} / \mathrm{dL}$ (15-40

'Department of Paediatric Neurology; ${ }^{2}$ Department of Paediatric Radiology; ${ }^{3}$ Department of Paediatrics, Dokuz Eylül University School of Medicine, Izmir, Turkey (Correspondence to U. Yis: ulyis@yahoo.com).

Received: 18/06/06; accepted: 06/08/06 


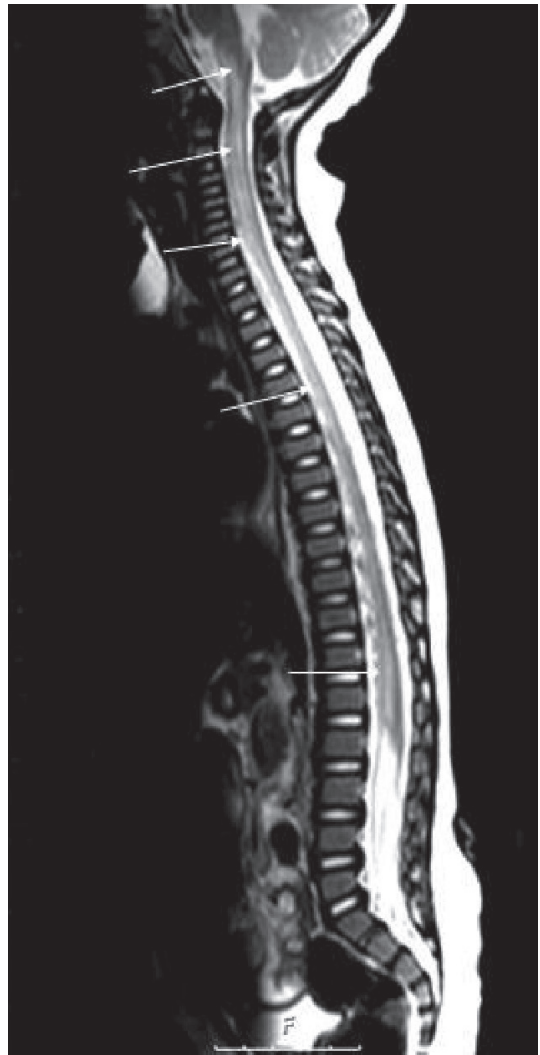

Figure 1 T2 weighted sagittal magnetic resonance image showing increased signal within the whole spinal cord and medulla oblongata

$\mathrm{mg} / \mathrm{dL}$ is the normal range for cerebrospinal fluid protein in our laboratory) and glucose content was $58 \mathrm{mg} / \mathrm{dL}$ (simultaneous blood sugar $90 \mathrm{mg} / \mathrm{dL}$ ). There was no pleocytosis. No malignant cells were seen in the cerebrospinal fluid. Cerebrospinal fluid anti-GM1 ganglioside antibodies and oligoclonal band were negative and the IgG index was normal. Serum and cerebrospinal fluid serological analysis_-including herpes simplex 1 and 2, varicella zoster, cytomegalovirus, Epstein-Barr virus, rubella, measles, mumps, mycoplasma and enterovirus - demonstrated no abnormalities. Electromyography was normal.

The patient was diagnosed with diffuse myelitis and treated at first with intravenous (IV) immunoglobulin $(400 \mathrm{mg} / \mathrm{kg} /$ day for 5 days). Response to IV immunoglobulin treatment was delayed for 1 week but because of insufficient remission in his clinical condition, IV pulse methylprednisolone (30 $\mathrm{mg} / \mathrm{kg} /$ day for 3 days) was started. On day 5 of steroid treatment there was a marked improvement in head control and on day 10 of steroid treatment the patient was able to sit and move his upper and lower limbs

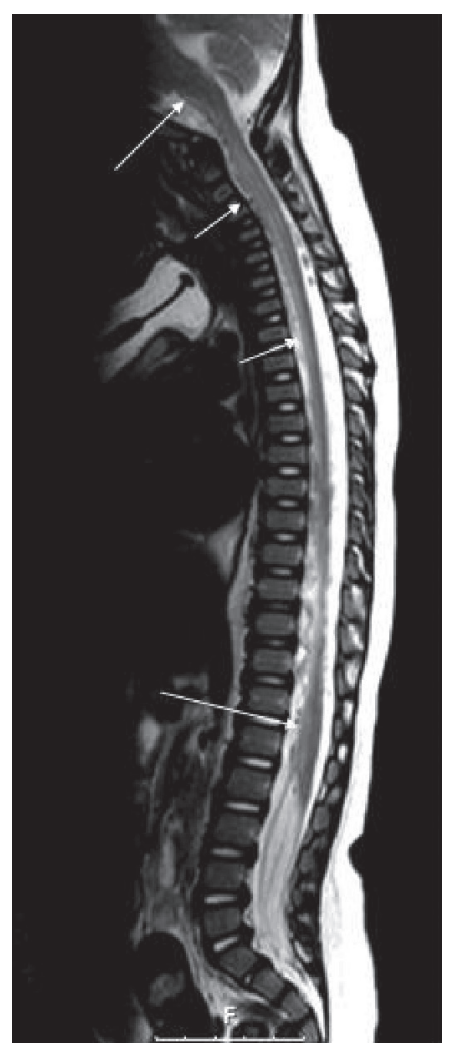

Figure 2 Control magnetic resonance imaging demonstrating decreased lesion signal in the involved spinal cord

المجلة الصحية لشرق المتوسط، منظمة الصحة العالمية، المجلد الخامس عشر، العدد (، 9 ·. 
independently. Post-treatment magnetic resonance imaging demonstrated decreased lesion signals in the involved spinal cord (Figure 2).

\section{Discussion}

The differential diagnosis of an acute disease of the spinal cord includes many conditions [6,7]. Bacterial abscesses, spinal cord tumours, vascular malformations and haematomas can be excluded with neuroimaging methods. In the present case the symptoms and signs included quadriparesis and areflexia. These findings were typical of spinal cord syndrome, warranting urgent imaging of the cord to exclude a space-occupying lesion such as a neoplasm, vascular malformation or haemorrhage. Cranial neuroimaging is also necessary to rule out cerebral involvement, as occurs in multiple sclerosis or acute disseminated encephalomyelitis [8]. Furthermore, immunologic disease of the spinal cord may not be confined to the spinal cord, but may also involve the motor neurons and/or the peripheral nerves, and for this reason every case should be evaluated with electromyography. Clinical, radiological and electrophysiological findings in this patient showed isolated diffuse myelitis.

Acute transverse myelitis affects all ages except children under 2 years. The present case was 9 months old and to our knowledge he is the one of the youngest cases in the literature with diffuse, symmetrical spinal cord involvement.

Three pathophysiological mechanisms seem to be responsible in the etiology of acute transverse myelitis: direct infection of the spinal cord; myelitis because of a systemic disease such as leukaemia or a connective tissue disorder; and autoimmunity $[9,10]$. The last group is the most common form of myelitis and may occur as a post- or parainfectious condition [11]. In $30 \%-60 \%$ of idiopathic myelitis cases there is an antecedent respiratory, gastrointestinal or systemic illness. On the other hand, several reports of myelitis and central nervous system demyelinating lesions following vaccination with for example influenza and hepatitis $B$ have recently been published $[12,13]$. It is likely that there is abnormal activation of the immune system resulting in inflammation and injury within the spinal cord. Autopsy evaluation of the spinal cord has revealed severe axonal loss with mild demyelination and a mononuclear infiltrate, predominantly $\mathrm{T}$ lymphocytes in the nerve roots and spinal ganglia. It is possible that the concomitant exposition of 2 different antigens (viral respiratory infection and immunization) increase the risk of an abnormal immune-mediated response in genetically susceptible individuals [14-17]. The patient had no evidence of a systemic infectious disease but he had a history of a viral respiratory infection. It is probable that this child had post-infectious myelitis as the neurological symptoms followed the infection by 3 weeks.

While some reports found high-dose methylprednisolone to be effective in acute transverse myelitis, some found no difference between patients given prednisolone, highdose methylprednisolone or gammaglobulin $[18,19]$. The present case was treated as first step with IV immunoglobulin because of the undesired adverse effects of steroids in this age group. Although the response to IV immunoglobulin may not appear directly after treatment, and may be delayed days or weeks, IV methylprednisolone was started because of poor remission and to prevent a worse outcome such as respiratory insufficiency. The response to steroid treatment was excellent and the patient recovered rapidly in the first week of steroid treatment. Regardless of age, patients should 
be treated as a first step with steroids and if there is no or insufficient response, IV immunoglobulin, plasmapheresis or other immunosuppressive drugs should be tried as alternative treatments.

The outcome of acute transverse myelitis among childhood cases has been cited in many cases as "good" and despite reports of fatalities, outcome is considered to be better for children than adults $[3,6]$. Recovery, if it occurs, should begin within 6 months, and most patients begin to show some restoration of neurological function within 8 weeks [20]. Our findings do not support the MRI evidence of diffuse myelopathy, such as hyperintensity on $\mathrm{T} 2-\mathrm{W}$ images, patchy enhancement and contrast enhancement (which are important prognostic indicators of poor outcome in childhood myelitis [21]), since the case recovered quickly after the steroid treatment despite diffuse involvement of the spinal cord. However, in keeping with previous reports, the present case supports the findings of Andronikou et al. who demonstrated 3 cases in which the extensive spinal cord hyperintensity on $\mathrm{T} 2-\mathrm{W}$ images did not correlate with the severity of the clinical presentation or outcome [22].

In conclusion, acute transverse myelitis is a rare but severe inflammatory demyelinating disorder of the spinal cord. It is very rare in the paediatric population, especially children under 2 years of age. Although the involvement of the spinal cord in acute transverse myelitis is segmental, children may present with entire spinal involvement. In the differential diagnosis of acquired hypotonia in infants, myelitis should always be considered and immediate MRI of the spine must be done to reach a diagnosis.

\section{References}

1. Tartaglino LM et al. Idiopathic acute transverse myelitis: MR imaging findings. Radiology, 1996, 201:661-8.

2. Murthy JM et al. Acute transverse myelitis: MR characteristics. Neurology India, 1999, 47:290-3.

3. Lahat $\mathrm{E}$ et al. Rapid recovery from transverse myelopathy in children treated with methylprednisolone. Pediatric neurology, 1998, 19:279-82.

4. De Seze J et al. Idiopathic acute transverse myelitis: application of the recent diagnostic criteria. Neurology, 2005, 65:1950-3.

5. Mihai C, Jubelt B. Post-infectious encephalomyelitis. Current neurology and neuroscience reports, 2005, 5:440-5.

6. Knebusch M, Strassburg HM, Reiners K. Acute transverse myelitis in childhood: nine cases and review of the literature.
Developmental medicine and child neurology, 1998, 40:631-9.

7. Dunne K, Hopkins IJ, Shield LK. Acute transverse myelopathy in childhood. Developmental medicine and child neurology, 1986, 28:198-204.

8. Austin SG, Zee C-S, Waters C. The role of magnetic resonance imaging in acute transverse myelitis. Canadian journal of neurological sciences, 1992, 19:508-11.

9. Salgado CD, Weisse ME. Transverse myelitis associated with probable cat-scratch disease in a previously healthy pediatric patient. Clinical infectious diseases, 2000, 31:609-11.

10. Yavuz H, Cakir M. Transverse myelopathy: an initial presentation of acute leukemia. Pediatric neurology, 2001, 24:382-4.

11. Jeffery DR, Mandler RN, Davis LE. Transverse myelitis: retrospective analysis of 
33 cases, with differentiation of cases associated with multiple sclerosis and parainfectious events. Archives of neurology, 1993, 50:532-5.

12. Larner AJ, Farmer SF. Myelopathy following influenza vaccination in inflammatory CNS disorder treated with chronic immunosuppression. European journal of neurology, 2000, 7:731-3.

13. Sindern E et al. Inflammatory polyradiculoneuropathy with spinal cord involvement and lethal outcome after hepatitis B vaccination. Journal of the neurological sciences, 2001, 186:81-5.

14. Monteyne $\mathrm{P}$, Andre FE. Is there a causal link between hepatitis $B$ vaccination and multiple sclerosis? Vaccine, 2000, 18:1994-2001.

15. Merelli E, Casoni F. Prognostic factors in multiple sclerosis: role of intercurrent infections and vaccinations against influenza and hepatitis B. Neurological sciences, 2000, 21:853-6.

16. Moriabadi NF et al. Influenza vaccination in MS: absence of T-cell response against white matter proteins. Neurology, 2001, 56:938-43.
17. Confavreux $\mathrm{C}$ et al. Vaccinations and the risk of relapse in multiple sclerosis. Vaccines in Multiple Sclerosis Study Group. New England journal of medicine, 2001, 344:319-26.

18. Sebire $G$ et al. High dose methylprednisolone in severe acute transverse myelopathy. Archives of disease in childhood, 1997, 76:167-8.

19. Defresne P et al. Efficacy of high dose steroid therapy in children with severe acute transverse myelitis. Journal of neurology, neurosurgery, and psychiatry, 2001, 71:272-4.

20. Pandit L, Rao S. Recurrent myelitis. Journal of neurology, neurosurgery, and psychiatry, 1996, 60:336-8.

21. Scott $T$ et al. Acute transverse myelitis: a retrospective study using magnetic resonance imaging. Canadian journal of neurological sciences, 1994, 21:133-6.

22. Andronikou $S$ et al. MRI findings in acute idiopathic transverse myelopathy in children. Pediatric radiology, 2003, 33:624-9. 